\title{
Reverse lexicographic and lexicographic shifting
}

\author{
Eric Babson · Isabella Novik • Rekha Thomas
}

Received: August 26, 2004 / Revised: June 14, 2005 / Accepted: July 5, 2005

(C) Springer Science + Business Media, Inc. 2006

\begin{abstract}
A short new proof of the fact that all shifted complexes are fixed by reverse lexicographic shifting is given. A notion of lexicographic shifting, $\Delta_{\text {lex }}$-an operation that transforms a monomial ideal of $S=\mathbf{k}\left[x_{i}: i \in \mathbb{N}\right]$ that is finitely generated in each degree into a squarefree strongly stable ideal-is defined and studied. It is proved that (in contrast to the reverse lexicographic case) a squarefree strongly stable ideal $I \subset S$ is fixed by lexicographic shifting if and only if $I$ is a universal squarefree lexsegment ideal (abbreviated USLI) of $S$. Moreover, in the case when $I$ is finitely generated and is not a USLI, it is verified that all the ideals in the sequence $\left\{\Delta_{\text {lex }}^{i}(I)\right\}_{i=0}^{\infty}$ are distinct. The limit ideal $\bar{\Delta}(I)=\lim _{i \rightarrow \infty} \Delta_{\text {lex }}^{i}(I)$ is well defined and is a USLI that depends only on a certain analog of the Hilbert function of $I$.
\end{abstract}

Keywords Shifting $\cdot$ Reverse lexicographic

\section{Introduction}

This paper deals with two problems related to algebraic shifting that were raised by Gil Kalai in [15].

Algebraic shifting is an algebraic operation introduced by Kalai $[6,14]$ that transforms a simplicial complex $\Gamma$ into a simpler (shifted) complex $\Delta(\Gamma)$, while preserving important combinatorial, topological and algebraic invariants such as face numbers, reduced Betti numbers and extremal algebraic Betti numbers. There are two versions of algebraic shifting-exterior and symmetric: the first one amounts to computing the (degree) reverse lexicographic generic initial ideal $\left(\mathrm{Gin}_{\mathrm{rl}}\right)$ of the Stanley-Reisner ideal of $\Gamma$ in the exterior algebra, while the second one amounts to computing $\mathrm{Gin}_{\mathrm{rl}}$ in the symmetric algebra and then applying a certain "squarefree" operation $\Phi$. In this paper we consider only the symmetric version of algebraic shifting. We refer to this operation as revlex shifting and denote it by $\Delta_{\mathrm{rl}}$.

Research partially supported by NSF grants DMS 0070571 and DMS 0100141

E. Babson $(\bowtie) \cdot$ I. Novik · R. Thomas

Department of Mathematics, University of Washington, Seattle, WA 98195-4350

email: $\{$ babson, novik, thomas $\} @$ math.washington.edu 
Clearly $\Delta_{\mathrm{rl}}(\Gamma) \neq \Gamma$ if $\Gamma$ is not shifted. Among the many beautiful properties of revlex shifting is the fact that the converse statement holds as well, namely that

$$
\Delta_{\mathrm{rl}}(\Gamma)=\Gamma \quad \text { if } \Gamma \text { is shifted, }
$$

and hence that $\Delta_{\mathrm{rl}}\left(\Delta_{\mathrm{rl}}(\Gamma)\right)=\Delta_{\mathrm{rl}}(\Gamma)$ for an arbitrary complex $\Gamma$. This result was stated in [14] and a somewhat hard proof was given in [3]. Equation (1) along with the two problems on algebraic shifting posed by Gil Kalai [15, Problems $16 \& 5$ ] is the starting point of our paper.

In [15, Problem 16] Kalai asks if algebraic shifting can be axiomatized. In that direction we prove the following result. (We denote by $[n]$ the set $\{1,2, \ldots, n\}$, and by $f(\Gamma)$ and $\beta_{i}(\Gamma)$, $i \geq 0$, the $f$-vector and the reduced simplicial Betti numbers of $\Gamma$ computed with coefficients in a field $\mathbf{k}$, respectively.)

Theorem 1.1. Let $\Delta$ be an operation that associates with every $n \geq 0$ and every simplicial complex $\Gamma$ on the vertex set $V=[n]$ a shifted simplicial complex $\Delta(\Gamma)$ on the same vertex set. Assume further that $\Delta$ satisfies the following properties:

(1) $f(\Delta(\Gamma))=f(\Gamma)$;

(2) $\Delta(\Gamma *\{n+1\})=\Delta(\Gamma) *\{n+1\}$;

(3) if $\Gamma^{\prime} \subseteq \Gamma$, then $\Delta\left(\Gamma^{\prime}\right) \subseteq \Delta(\Gamma)$;

(4) $\sum_{i=0}^{\operatorname{dim} \bar{\Gamma}} \beta_{i}(\Gamma) \leq \sum_{i=0}^{\operatorname{dim} \overline{\Delta(\Gamma)}} \beta_{i}(\Delta(\Gamma))$.

Then for every shifted complex $\Gamma, \Delta(\Gamma)=\Gamma$.

As a corollary we obtain a new and much simpler proof of Eq. (1). (Here $\Gamma *\{n+1\}$ is the cone over $\Gamma$, that is, a simplicial complex on the vertex set $[n+1]$ whose set of faces consists of faces of $\Gamma$ together with $\{F \cup\{n+1\}: F \in \Gamma\}$.)

Problem 5 in [15] asks whether the property given by Eq. (1) holds if one considers symmetric shiftings with respect to arbitrary term orders. Since in the case of exterior shiftings the answer is positive (as was shown by Kalai [13, Prop. 4.2]), one may expect to have the same result in the symmetric case as well. Here we consider (degree) lexicographic order, and denote the corresponding shifting operation by $\Delta_{\text {lex }}$. To our surprise we discover that only very few shifted complexes are fixed by lex shifting. Our results are summarized in Theorem 1.2 below.

Denote by $\mathbb{N}$ the set of all positive integers. We say that an ideal $I \subset S=\mathbf{k}\left[x_{i}: i \in \mathbb{N}\right]$ is a universal squarefree lexsegment ideal (abbreviated USLI) if it is finitely generated in each degree and is a squarefree lexsegment ideal of $S$. (Equivalently, an ideal $I$ of $S$ that is finitely generated in each degree is a USLI if $I \cap S_{[n]}$ is a squarefree lexsegment ideal of $S_{[n]}:=\mathbf{k}\left[x_{1}, \ldots, x_{n}\right]$ for every $n$.) Thus, for example, the ideal $\left\langle x_{1} x_{2}, x_{1} x_{3}, x_{1} x_{4} x_{5} x_{6} x_{7}\right\rangle$ is a USLI, while the ideal $\left\langle x_{1} x_{2}, x_{1} x_{3}, x_{2} x_{3}\right\rangle$ is a squarefree lexsegment of $S_{[3]}$ but is not a squarefree lexsegment of $S$, and hence is not a USLI. A simplicial complex $\Gamma$ is a USLI complex if its Stanley-Reisner ideal, $I_{\Gamma}$, is a USLI.

Recall that for a monomial ideal $J \subset S_{[n]}$ the (bi-graded) Betti numbers of $J$ are the invariants $\beta_{i, j}(J)$ that appear in the minimal free resolution of $J$ as an $S_{[n]}$-module.

$$
\cdots \bigoplus_{j} S_{[n]}(-j)^{\beta_{i, j}(J)} \rightarrow \cdots \rightarrow \bigoplus_{j} S_{[n]}(-j)^{\beta_{1, j}(J)} \rightarrow \bigoplus_{j} S_{[n]}(-j)^{\beta_{0, j}(J)} \rightarrow J \rightarrow 0
$$


Here $S_{[n]}(-j)$ denotes $S_{[n]}$ with grading shifted by $j$. Following [9], we define the $B$-sequence of $J, B(J):=\left\{B_{j}(J): j \geq 1\right\}$, where $B_{j}(J):=\sum_{i=0}^{j}(-1)^{i} \beta_{i, j}(J)$. (The $B$-sequence of an ideal contains the same information as its Hilbert series-see Section 5 for more details as well as for the definition of the $B$-sequence for a monomial ideal of $S$ that is finitely generated in each degree.)

\section{Theorem 1.2.}

(1) (finite) shifted simplicial complex $\Gamma$ satisfies $\Delta_{\operatorname{lex}}(\Gamma)=\Gamma$ if and only if $\Gamma$ is a USLI complex. Moreover, if $\Gamma$ is not a USLI complex, then all the complexes in the sequence $\left\{\Delta_{\text {lex }}^{i}(\Gamma)\right\}_{i=0}^{\infty}$ are distinct. (Here $\Delta_{\text {lex }}^{i}(\Gamma)$ denotes the complex obtained from $\Gamma$ by $i$ consecutive applications of $\Delta_{\mathrm{lex}}$.)

(2) The limit complex $\bar{\Delta}_{\text {lex }}(\Gamma):=\lim _{i \rightarrow \infty} \Delta_{\text {lex }}^{i}(\Gamma)$ is well defined and is a (usually infinite) USLI complex. Moreover, $\bar{\Delta}_{\text {lex }}(\Gamma)$ is the unique USLI complex whose Stanley-Reisner ideal has the same $B$-sequence as $I_{\Gamma}$.

The last part of the theorem implies that if two simplicial complexes $\Gamma_{1}$ and $\Gamma_{2}$ that have the same $h$-vector (up to possibly several zeros appended at the end), then $\bar{\Delta}_{\text {lex }}\left(\Gamma_{1}\right)=\bar{\Delta}_{\text {lex }}\left(\Gamma_{2}\right)$. Thus, in contrast to revlex shifting, the operation $\bar{\Delta}_{\text {lex }}$ forgets all the information that $\Gamma$ carries (including the dimension of $\Gamma$ ) except its $h$-numbers.

Our theorems establish for simplicial complexes, results similar in spirit to those in commutative algebra due to Bigatti-Conca-Robbiano [5] and Pardue [17]. Theorem 4.3 in [5] asserts that if $I$ is a strongly stable ideal in $S_{[n]}$ and $\mathcal{L}$ is a distraction matrix, then $\operatorname{Gin}_{\mathrm{rl}}\left(D_{\mathcal{L}}(I)\right)=I$, while Proposition 30 in [17] asserts that sufficiently (but finitely) many applications of the operation $\operatorname{Gin}_{\text {lex }} \circ D_{\mathcal{L}}$ to a monomial ideal $I \subset S_{[n]}$ results in the unique lexsegment ideal of $S_{[n]}$ having the same Hilbert function as $I$.

The structure of the paper is as follows. Section 2 is devoted to the proof of Theorem 1.1. In Section 3 after recalling basic facts and definitions related to generic initial ideals and revlex shifting we provide a short new proof of Eq. (1). In Section 4 we introduce and study the class of universal squarefree lexsegment ideals (USLIs) and the class of almost USLIs - the notions that play a crucial role in the proof of Theorem 1.2. Finally in Section 5 we prove Theorem 1.2. We close with a brief discussion of arbitrary term orders.

\section{Axiomatizing algebraic shifting}

This section is devoted to the proof of Theorem 1.1. We start by reviewing several notions pertaining to simplicial complexes.

Denote the collection of all subsets of $[n]:=\{1,2, \ldots, n\}$ by $2^{[n]}$. Recall that a simplicial complex $\Gamma$ on the vertex set $[n]$ is a collection $\Gamma \subseteq 2^{[n]}$ that is closed under inclusion. (We do not require that every singleton $\{i\} \subseteq[n]$ is an element of $\Gamma$.) The elements of $\Gamma$ are called faces and the maximal faces (under inclusion) are called facets. $F \in \Gamma$ is an $i$-dimensional face (or an $i$-face) if $|F|=i+1$. The dimension of $\Gamma$, $\operatorname{dim} \Gamma$, is the maximal dimension of its faces. The number of $i$-dimensional faces of $\Gamma$ is denoted by $f_{i}(\Gamma)$, and the sequence $f(\Gamma):=\left(f_{-1}(\Gamma), f_{0}(\Gamma), f_{1}(\Gamma), \ldots f_{\operatorname{dim} \Gamma}(\Gamma)\right)$ is called the $f$-vector of $\Gamma$. Another set of invariants associated with $\Gamma$ is the set of its reduced Betti numbers $\beta_{i}(\Gamma):=\operatorname{dim}_{\mathbf{k}} \tilde{H}_{i}(\Gamma ; \mathbf{k})$, where $\tilde{H}_{i}(\Gamma ; \mathbf{k})$ is the $i$-th reduced simplicial homology of $\Gamma$ with coefficients in a field $\mathbf{k}$.

A simplicial complex $\Gamma$ on the vertex set $[n]$ is called shifted if for every $F \in \Gamma, i \in F$, and $i<j \leq n$, the set $(F \backslash\{i\}) \cup\{j\}$ is a face of $\Gamma$ as well. The Betti numbers of a shifted 
complex $\Gamma$ are combinatorial invariants and can be computed via the following well-known formula [6, Thm. 4.3]:

Lemma 2.1. If $\Gamma$ is a shifted complex on the vertex set [ $n]$, then

$$
\beta_{i}(\Gamma)=|\{F \in \max (\Gamma):|F|=i+1, n \notin \Gamma\}|,
$$

where $\max (\Gamma)$ denotes the set of facets of $\Gamma$.

For a simplicial complex $\Gamma$ and a vertex $v$ of $\Gamma$ define the antistar of $v$ in $\Gamma$ as ast $\operatorname{ar}_{\Gamma}(v)=$ $\{F \in \Gamma: v \notin F\}$. Define also the link of $v$ in $\Gamma$ by $\operatorname{lk}_{\Gamma}(v):=\left\{F \in \operatorname{ast}_{\Gamma}(v): F \cup\{v\} \in \Gamma\right\}$. Note that if $\Gamma$ is a shifted complex on the vertex set $[n]$, then $\operatorname{lk}_{\Gamma}(n)$ and ast $\Gamma_{\Gamma}(n)$ are shifted complexes on $[n-1]$.

If $\Gamma$ is a simplicial complex on $V$ and $u \notin V$, then the cone over $\Gamma$ with apex $u$ is a simplicial complex, denoted $\Gamma *\{u\}$, on the vertex set $V \cup\{u\}$ whose faces are all sets of the form $F \cup A$, where $F \in \Gamma$ and $A \subseteq\{u\}$. Thus for any vertex $v$ of $\Gamma, \Gamma=\operatorname{lk}_{\Gamma}(v) *\{v\} \cup \operatorname{ast}_{\Gamma}(v)$ and $\mathrm{lk}_{\Gamma}(v) *\{v\} \subseteq \Gamma \subseteq \operatorname{ast}_{\Gamma}(v) *\{v\}$.

Now we are ready to verify Theorem 1.1 asserting that if $\Delta$ is an operation that associates with every $n \geq 0$ and every simplicial complex $\Gamma$ on the vertex set $V=[n]$ a shifted simplicial complex $\Delta(\Gamma)$ on the same vertex set, and if $\Delta$ satisfies the following properties:

1. $f(\Delta(\Gamma))=f(\Gamma)$;

2. $\Delta(\Gamma *\{n+1\})=\Delta(\Gamma) *\{n+1\}$

3. if $\Gamma^{\prime} \subseteq \Gamma$, then $\Delta\left(\Gamma^{\prime}\right) \subseteq \Delta(\Gamma)$;

4. $\sum_{i=0}^{\operatorname{dim}} \overline{\bar{\Gamma}} \beta_{i}(\Gamma) \leq \sum_{i=0}^{\operatorname{dim}} \overline{\Delta(\Gamma)} \beta_{i}(\Delta(\Gamma))$,

then for every shifted complex $\Gamma, \Delta(\Gamma)=\Gamma$.

Proof of Theorem 1.1: Fix a shifted complex $\Gamma$ on $n$ vertices. If $n=0$ or $n=1$ then $\Delta(\Gamma)=\Gamma$ by property (1). We proceed by induction on $n$. Since the link and the antistar of the vertex $n$ in $\Gamma, \Gamma^{\prime}=\operatorname{lk}_{\Gamma}(n)$ and $\Gamma^{\prime \prime}=\operatorname{ast}_{\Gamma}(n)$, respectively, are shifted complexes on the vertex set $[n-1]$ and since $\Gamma^{\prime} *\{n\} \subseteq \Gamma \subseteq \Gamma^{\prime \prime} *\{n\}$, the induction hypothesis together with properties (2) and (3) yield

$$
\Gamma^{\prime} *\{n\} \subseteq \Delta(\Gamma) \subseteq \Gamma^{\prime \prime} *\{n\}
$$

Therefore,

$$
\begin{aligned}
A: & =\{F \in \max (\Delta(\Gamma)): n \notin F\}=\left\{F \in \max (\Delta(\Gamma)): F \in \Gamma^{\prime \prime}\right\} \\
& \subseteq \Gamma^{\prime \prime} \backslash \Gamma^{\prime} \stackrel{(\star)}{=}\{F \in \max (\Gamma): n \notin F\}=: B,
\end{aligned}
$$

where $(\star)$ follows from the shiftedness of $\Gamma$ :

$F \in \max (\Gamma) \cap 2^{[n-1]} \Longleftrightarrow F \in \Gamma$ but $F \cup\{n\} \notin \Gamma \Longleftrightarrow F \in \operatorname{ast}_{\Gamma}(n) \backslash \operatorname{lk}_{\Gamma}(n)=\Gamma^{\prime \prime} \backslash \Gamma^{\prime}$.

On the other hand, Lemma 2.1 and property (4) imply that

$$
|A|=\sum_{i=0}^{\operatorname{dim} \Delta(\Gamma)} \beta_{i}(\Delta(\Gamma)) \geq \sum_{i=0}^{\operatorname{dim} \Gamma} \beta_{i}(\Gamma)=|B|,
$$


and thus that $A=B$. Hence $\Delta(\Gamma) \supseteq A=\Gamma^{\prime \prime} \backslash \Gamma^{\prime}$, and we infer that

$$
\Delta(\Gamma) \supseteq\left(\Gamma^{\prime} *\{n\}\right) \cup\left(\Gamma^{\prime \prime} \backslash \Gamma^{\prime}\right)=\Gamma .
$$

Since $f(\Gamma)=f(\Delta(\Gamma))$ by property (1), it follows that $\Delta(\Gamma)=\Gamma$.

\section{Generic initial ideals and revlex shifting}

In this section we review basic facts and definitions related to generic initial ideals and revlex shifting. We also provide a new short proof of Eq. (1) asserting that $\Delta_{\mathrm{rl}}(\Gamma)=\Gamma$ for a shifted $\Gamma$. Let $S_{[n]}=\mathbf{k}\left[x_{1}, \ldots, x_{n}\right]$ be the ring of polynomials in $n$ variables over a field $\mathbf{k}$ of characteristic zero, and let $\Gamma$ be a simplicial complex on the vertex set $[n]$. We recall that the Stanley-Reisner ideal of $\Gamma[18]$ is the squarefree monomial ideal $I_{\Gamma} \subset S_{[n]}$ whose generators correspond to nonfaces of $\Gamma$ :

$$
I_{\Gamma}:=\left\langle\prod_{j=1}^{k} x_{i_{j}} \in S_{[n]}:\left\{i_{1}<i_{2}<\cdots<i_{k}\right\} \notin \Gamma\right\rangle .
$$

The Stanley-Reisner ideal of a shifted complex is called a squarefree strongly stable ideal. (Equivalently, a squarefree monomial ideal $I$ is squarefree strongly stable, if for every minimal generator $m$ of $I$ and for every $1 \leq i<j$ such that $x_{j} \mid m$ but $x_{i} \backslash \chi m$, the monomial $m x_{i} / x_{j}$ lies in $I$.)

Let $\succ$ be a term order on $S_{[n]}$ that refines the partial order by degree where lower degree monomials are more expensive than higher degree monomials, and satisfies $x_{1} \succ \cdots \succ x_{n}$. Let $I \subset S_{[n]}$ be a homogeneous ideal such as the Stanley-Reisner ideal of $\Gamma$. Consider a generic $n \times n$ matrix $g$. Then $g$ acts on the set of linear forms of $S_{[n]}$ by $g x_{j}=\sum_{i=1}^{n} g_{i j} x_{i}$ and this action can be extended uniquely to a ring automorphism on $S_{[n]}$ that we also denote by $g$. Following [8, Thm. 15.18] define the generic initial ideal of $I$ with respect to $\succ$ as

$$
\operatorname{Gin}_{\succ}(I):=\operatorname{in}_{\succ}(g I)
$$

where in $\succ(g I)$ is the initial ideal of $g I$ with respect to $\succ$ in the sense of Gröbner basis theory. The same theorem in [8] asserts that we can choose $g$ to be upper triangular and hence we assume from now on that $g x_{j}=\sum_{i=1}^{j} g_{i j} x_{i}$.

We briefly outline how to compute $\operatorname{Gin}_{\succ}(I)$ (for a detailed description the reader is referred to $[8$, Thm. 15.18]).

Definition 3.1. An exterior monomial in $\bigwedge^{l}\left(S_{[n]}\right)_{d}$ is an element of the form $m_{1} \wedge \cdots \wedge m_{l}$ where each $m_{i}$ is a monomial of $S_{[n]}$ of degree $d$ and $m_{1} \succ \cdots \succ m_{l}$. The extension of $\succ$ to an order on monomials of $\bigwedge^{l}\left(S_{[n]}\right)_{d}$ is the order in which $m_{1} \wedge \cdots \wedge m_{l} \succ n_{1} \wedge \cdots \wedge n_{l}$ if for some $s$ we have that $m_{s} \succ n_{s}$ and $m_{i}=n_{i}$ for $i<s$. For a non-zero element $f$ of $\bigwedge^{l}\left(S_{[n]}\right)_{d}$, define the initial term of $f, \operatorname{in}_{\succ}(f)$, to be the $\succ$-largest monomial appearing in $f$ with nonzero coefficient when $f$ is written as a linear combination of (distinct) monomials.

Consider a generic $n \times n$ upper-triangular matrix $g$ and its action on $S_{[n]}$. Let $I_{d}$ be the $d$-th homogeneous component of a homogeneous ideal $I$, and let $f_{1}, \ldots, f_{t}$ be a basis 
of $I_{d}$. Then $g\left(f_{1}\right) \wedge \cdots \wedge g\left(f_{t}\right) \in \wedge^{t}\left(S_{[n]}\right)_{d}$. Denote by $M_{d}=m_{1} \wedge \cdots \wedge m_{t}$ the monomial $\operatorname{in}_{\succ}\left(g\left(f_{1}\right) \wedge \cdots \wedge g\left(f_{t}\right)\right)$ and by $V_{d}$ the subspace of $\left(S_{[n]}\right)_{d}$ spanned by $m_{1}, \ldots, m_{t}$.

Proposition 3.2. $\operatorname{Gin}_{\succ}(I)=\bigoplus V_{d}$.

Several basic properties of Gins are summarized in the following lemma.

Lemma 3.3. Let $I \subset S_{[n]}$ be a homogeneous ideal. Then

(1) $\operatorname{Gin}_{\succ}(I)$ is a strongly stable monomial ideal (that is, if $m \in \operatorname{Gin}_{\succ}(I), x_{j} \mid m$ and $1 \leq i<j$, then $x_{i} m / x_{j} \in \operatorname{Gin}_{\succ}(I)$ as well).

(2) $\operatorname{Gin}_{\succ}(I)$ and I have the same Hilbert function (that is, $\operatorname{dim}_{\mathbf{k}}\left(\operatorname{Gin}_{\succ}(I)_{d}\right)=\operatorname{dim}_{\mathbf{k}}\left(I_{d}\right)$ for all $d)$.

(3) If $J \subseteq I$ is a homogeneous ideal of $S_{[n]}$, then $\operatorname{Gin}_{\succ}(J) \subseteq \operatorname{Gin}_{\succ}(I)$.

(4) Let $\succ^{\prime}$ be an extension of $\succ$ to a term order on $S_{[n+1]}$ satisfying $x_{n} \succ^{\prime} x_{n+1}$. Then $\operatorname{Gin}_{\succ^{\prime}}\left(I S_{[n+1]}\right)=\left(\operatorname{Gin}_{\succ} I\right) S_{[n+1]}$. In particular, for a simplicial complex $\Gamma$ on $[n]$, $\operatorname{Gin}_{\succ^{\prime}}\left(I_{\Gamma *\{n+1\}}\right)=\left(\operatorname{Gin}_{\succ} I_{\Gamma}\right) S_{[n+1]}$.

Proof: Part (1) is [8, Thm. 15.18 and Thm. 15.23]. Part (2) follows from [8, Thm 15.3]. Part (3) is obvious from the definitions. To prove part (4), consider a generic upper-triangular $(n+1) \times(n+1)$ matrix $\tilde{g}$ and its left-upper $n \times n$ submatrix $g$. Then $g$ acts on $S_{[n]}, \tilde{g}$ acts on $S_{[n+1]}$, and $\tilde{g} x_{i}=g x_{i}$ for all $1 \leq i \leq n$. Therefore for every (homogeneous) element $h$ of $I \subset S_{[n]} \subset S_{[n+1]}, \tilde{g} h=g h$. Thus for $h \in I, \operatorname{in}_{\succ^{\prime}}(\tilde{g} h)=\operatorname{in}_{\succ}(g h)$, implying that $\operatorname{Gin}_{\succ} I \subseteq$ $\operatorname{Gin}_{\succ^{\prime}}\left(I S_{[n+1]}\right)$, and hence that $\left(\operatorname{Gin}_{\succ} I\right) S_{[n+1]} \subseteq \operatorname{Gin}_{\succ^{\prime}}\left(I S_{[n+1]}\right)$. The lemma follows, since both the ideals $\left(\operatorname{Gin}_{\succ} I\right) S_{[n+1]}$ and $\operatorname{Gin}_{\succ^{\prime}}\left(I S_{[n+1]}\right)$ have the same Hilbert function.

In the later sections we compare Gins of the same ideal $I$ computed with respect to different term orders. For that we need the following definition.

Definition 3.4. Let $I_{1} \neq I_{2}$ be two monomial ideals of $S_{[n]}$ and let $\succ$ be a term order. We say that $I_{1} \succ I_{2}$ if the largest monomial in the symmetric difference of $I_{1}$ and $I_{2}$ is in $I_{1}$. Equivalently, $I_{1} \succ I_{2}$ if the largest monomial in the symmetric difference of $G\left(I_{1}\right)$ and $G\left(I_{2}\right)$ is in $G\left(I_{1}\right)$, where $G\left(I_{1}\right)$ and $G\left(I_{2}\right)$ are the sets of minimal generators of $I_{1}$ and $I_{2}$ respectively.

One immediate observation is

Lemma 3.5. Let $\sigma$ and $\tau$ be two term orders on $S_{[n]}$. Then $\operatorname{Gin}_{\sigma}(I) \geq_{\sigma} \operatorname{Gin}_{\tau}(I)$ for any homogeneous ideal I $\subset S_{[n]}$.

Proof: Let $f_{1}, \ldots, f_{t}$ be a basis of $I_{d}$, and let $g$ be a generic $n \times n$ upper-triangular matrix. Since $M_{d}^{\prime}:=\operatorname{in}_{>_{\tau}}\left(g\left(f_{1}\right) \wedge \cdots \wedge g\left(f_{t}\right)\right)$ appears in $g\left(f_{1}\right) \wedge \cdots \wedge g\left(f_{t}\right)$ with a non-zero coefficient, it follows that $M_{d}:=\operatorname{in}_{\sigma}\left(g\left(f_{1}\right) \wedge \cdots \wedge g\left(f_{t}\right)\right) \geq_{\sigma} M_{d}^{\prime}$ (for every $d \geq 0$ ). Proposition 3.2 implies the lemma.

We remark that a stronger version of Lemma 3.5 was proved in [7, Cor. 1.6].

Another ingredient needed for defining revlex shifting is the notion of the squarefree operation. This is a bijection $\Phi$ between the set of all monomials in $\left\{x_{i}: i \in \mathbb{N}\right\}$ and the set 且 Springer 
of all squarefree monomials in $\left\{x_{i}: i \in \mathbb{N}\right\}$, defined by

$$
\Phi\left(\prod_{j=1}^{k} x_{i_{j}}\right)=\prod_{j=1}^{k} x_{i_{j}+j-1}, \quad \text { where } i_{1} \leq i_{2} \leq \cdots \leq i_{k} .
$$

Note that for a monomial $m \in S_{[n]}, \Phi(m)$ may not belong to $S_{[n]}$. However the graded reverse lexicographic order has the following remarkable property [14, Lemma 6.3(ii)], [3, Lemma 1.1]: if $m$ is a minimal generator of $\operatorname{Gin}_{\mathrm{rl}} I_{\Gamma}$ (where $\Gamma$ is a simplicial complex on $[n]$ ), then $\Phi(m)$ is an element of $S_{[n]}$. This leads to the following definition (due to Kalai).

Definition 3.6. Let $\Gamma$ be a simplicial complex on the vertex set $[n]$. The reverse lexicographic shifting of $\Gamma, \Delta_{\mathrm{rl}}(\Gamma)$, is a simplicial complex on $[n]$ whose Stanley-Reisner ideal is given by

$$
I_{\Delta_{\mathrm{rl}}(\Gamma)}=\left\langle\Phi(m): m \in G\left(\operatorname{Gin}_{\mathrm{rl}} I_{\Gamma}\right)\right\rangle
$$

where $G(I)$ denotes the set of the minimal generators of a monomial ideal $I$.

We now provide a new and simple proof of Eq. (1) (due originally to Aramova, Herzog, and Hibi [3]).

Theorem 3.7. The revlex shifting $\Delta_{\mathrm{rl}}$ satisfies all the conditions of Theorem 1.1. Thus $\Delta_{\mathrm{rl}}(\Gamma)=\Gamma$ for every shifted complex $\Gamma$.

Proof: It is well-known that (symmetric) revlex shifting satisfies all the conditions of Theorem 1.1, except possibly for property (2) whose proof appears to be missing in the literature (for the exterior version of algebraic shifting it was recently verified by Nevo [16]): the fact that $\Delta(\Gamma)$ is a shifted simplicial complex follows from Lemma 3.3(1); property (1) is [14, Lemma 6.3(i)]; property (3) is a consequence of Lemma 3.3(3); property (4) follows from [11, Cor. 8.25] asserting that $\beta_{i}(\Gamma)=\beta_{i}(\Delta(\Gamma))$ for all $i$. To prove property (2) it suffices to check that $\Delta(\Gamma)$ and $\Delta(\Gamma \star\{n+1\})$ have the same set of minimal nonfaces (equivalently, $I_{\Delta(\Gamma)} \subset S_{[n]}$ and $I_{\Delta(\Gamma \star\{n+1\})} \subset S_{[n+1]}$ have the same set of minimal generators). This follows from Definition 3.6 and Lemma 3.3(4).

\section{Remarks}

(1) We note that to verify the inequality $\sum \beta_{i}(\Gamma) \leq \sum \beta_{i}\left(\Delta_{\mathrm{rl}}(\Gamma)\right)$ one does not need to use the fact that $\beta_{i}(\Gamma)=\beta_{i}\left(\Delta_{\mathrm{rl}}(\Gamma)\right)$ for all $i$, which is a consequence of the deep result due to Bayer-Charalambous-Popescu [4] and Aramova-Herzog [1] that revlex shifting preserves extremal (algebraic) Betti numbers. Instead one can use the standard flatness argument (see [11, Thm. 3.1]) to show that $\beta_{i, j}\left(I_{\Gamma}\right) \leq \beta_{i, j}\left(\operatorname{Gin}_{\mathrm{rl}}\left(I_{\Gamma}\right)\right)=\beta_{i, j}\left(I_{\Delta(\Gamma)}\right)$ for all $i, j$, where the equality comes from the fact that $\Phi$ applied to (minimal generators of) a strongly stable ideal $\operatorname{Gin}_{\mathrm{rl}}\left(I_{\Gamma}\right)$ preserves algebraic Betti numbers (see [3, Lemma 2.2]). The Hochster formula [12] then asserts that the reduced Betti numbers of a simplicial complex are equal to certain algebraic graded Betti numbers of its Stanley-Reisner ideal.

(2) In algebraic terms, the statement of Theorem 3.7 translates to the fact that if $I \subset S_{[n]}$ is a squarefree strongly stable ideal, then $\Phi\left(\operatorname{Gin}_{\mathrm{rl}}(I)\right)=I$, where $\Phi\left(\operatorname{Gin}_{\mathrm{rl}}(I)\right):=\langle\Phi(m)$ : $\left.m \in G\left(\operatorname{Gin}_{\mathrm{rl}}(I)\right)\right\rangle$. Hence $\operatorname{Gin}_{\mathrm{rl}}(I)=\left\langle\Phi^{-1}(\mu): \mu \in G(I)\right\rangle$, that is, computing the revlex 
Gin of a squarefree strongly stable ideal $I$ simply amounts to applying $\Phi^{-1}$ to the minimal generators of $I$.

(3) Our proof (as well as the original proof in [3]) of the equation $\Phi\left(\operatorname{Gin}_{\mathrm{rl}}(I)\right)=I$ for a squarefree strongly stable ideal $I$ works only over a field $\mathbf{k}$ of characteristic zero. We however do not know of any counterexamples in the case of a field of positive characteristic.

\section{Combinatorics of USLIs, almost USLIs, and lex Gins}

In this section we introduce and study the class of universal squarefree lexsegment ideals (USLIs) and the class of almost USLIs. These notions turn out to be crucial in the proof of Theorem 1.2. To allow for infinitely generated ideals (as we need in the following section) we consider the system of rings $S_{[n]}, n \in \mathbb{N}$, endowed with natural embeddings $S_{[n]} \subseteq S_{[m]}$ for $m \geq n$, and provide definitions suitable for the direct limit ring $S=\lim _{n \rightarrow \infty} S_{[n]}=\mathbf{k}\left[x_{i}\right.$ : $i \in \mathbb{N}]$.

Recall that a squarefree monomial ideal $I \subset S$ ( $I \subset S_{[n]}$, respectively) is a squarefree lexsegment ideal of $S$ ( $S_{[n]}$, respectively) if for every monomial $m \in I$ and every squarefree monomial $m^{\prime} \in S\left(m^{\prime} \in S_{[n]}\right.$, respectively) such that $\operatorname{deg}\left(m^{\prime}\right)=\operatorname{deg}(m)$ and $m^{\prime}>_{\text {lex }} m, m^{\prime}$ is an element of $I$ as well.

Definition 4.1. An ideal $L$ of $S$ (or of $S_{[n]}$ ) is a universal squarefree lexsegment ideal (abbreviated USLI) if it is finitely generated in each degree and $L S$ is a squarefree lexsegment ideal of $S$. Equivalently, an ideal $L=L\left(k_{\bullet}\right)$ (here $k_{\bullet}=\left\{k_{i}\right\}_{i \in \mathbb{N}}$ is a sequence of nonnegative integers) is a USLI with $k_{i}$ minimal generators of degree $i$ (for $i \in \mathbb{N}$ ) if and only if the set of minimal generators of $L, G(L)$, is given by

$$
G(L)=\bigcup_{r=1}^{\infty}\left\{\left(\prod_{j=1}^{r-1} x_{R_{j}}\right) \cdot x_{l}: R_{r-1}+1 \leq l \leq R_{r}-1\right\}, \quad \text { where } R_{j}=j+\sum_{i=1}^{j} k_{i} .
$$

The easiest way to verify the description of the set $G(L)=\left\{m_{1}>_{\text {lex }} m_{2}>_{\text {lex }} \cdots>_{\text {lex }}\right.$ $\left.m_{s}>_{\text {lex }} \cdots\right\}$ of a USLI $L$ is by induction on $s$. Indeed, if $m_{1}, \ldots, m_{s}$ satisfy the above description and $m_{s}=\left(\prod_{j=1}^{r-1} x_{R_{j}}\right) \cdot x_{l}$, then there are two possibilities for $m_{s+1}$ : either $\operatorname{deg}\left(m_{s+1}\right)=\operatorname{deg}\left(m_{s}\right)=r$ (equivalently, $\left.l<R_{r}-1\right)$ or $\operatorname{deg}\left(m_{s+1}\right)=r^{\prime}>r$ (equivalently, $l=R_{r}-1$ and $k_{i}=0$ for all $\left.r<i<r^{\prime}\right)$. In the former case, since $m_{s}>_{\operatorname{lex}} m_{s+1}$ and since $m_{s}$ is the immediate lex-predecessor of $m^{\prime}:=\left(\prod_{j=1}^{r-1} x_{R_{j}}\right) \cdot x_{l+1}$, it follows that $m^{\prime} \geq_{\text {lex }} m_{s+1} \in L$ which together with $L$ being a USLI implies that $m^{\prime} \in L$. Since $m^{\prime}$ is not divisible by any of $m_{1}, \ldots, m_{s}$, this yields $m_{s+1}=m^{\prime}$. The treatment of the latter case is similar: just observe that every squarefree monomial of degree $r^{\prime}$ that is lex-smaller than $m^{\prime}:=\left(\prod_{j=1}^{r-1} x_{R_{j}}\right) \cdot\left(\prod_{j=1}^{r^{\prime}-r+1} x_{l+j}\right)=\left(\prod_{j=1}^{r^{\prime}-1} x_{R_{j}}\right) \cdot x_{R_{r^{\prime}-1}+1}$ is divisible by at least one of $m_{1}, \ldots, m_{s}$ and hence is in $L-G(L)$, while $m^{\prime}$ is not divisible by any of $m_{1}, \ldots, m_{s}$.

\section{Example 4.2.}

(1) The ideal $\left\langle x_{1} x_{2}, x_{1} x_{3}, x_{2} x_{3}\right\rangle$ (the Stanley-Reisner ideal of three isolated points) is a lexsegment in $S_{[3]}$, but is not a lexsegment in $S$, and hence is not a USLI. 
(2) The ideal $I=\left\langle x_{1} x_{2}, x_{1} x_{3}, x_{1} x_{4} x_{5} x_{6} x_{7}\right\rangle$ is the USLI with $k_{1}=0, k_{2}=2, k_{3}=k_{4}=$ $0, k_{5}=1$ and $k_{i}=0$ for all $i>5$. In this example, check that $R_{1}=1, R_{2}=4, R_{3}=$ $5, R_{4}=6$ and $R_{5}=8$.

Note that every USLI is a squarefree strongly stable ideal, and hence is the StanleyReisner ideal of a shifted (possibly infinite) simplicial complex (we refer to such complex as a USLI complex). All complexes considered in this section are assumed to be finite.

The following lemma describes certain combinatorial properties of USLI complexes. This lemma together with Lemmas 4.5 and 4.6 below provides a key step in the proof of Theorem 1.2.

Lemma 4.3. Let $\Gamma$ be a USLI complex on the vertex set $[n]$ with $I_{\Gamma}=L\left(k_{\bullet}\right)$.

(1) If $I_{\Gamma} \neq 0$ and $k_{d}$ is the last nonzero entry in the sequence $k_{\bullet}$, then $\Gamma$ has exactly $d$ facets. They are given by

$$
F_{i}= \begin{cases}\left\{R_{j}: 1 \leq j \leq i-1\right\} \cup\left[R_{i}+1, n\right] & \text { if } 1 \leq i \leq d-1, \\ \left\{R_{1}, \ldots, R_{d-1}\right\} \cup\left[R_{d}, n\right] & \text { if } i=d\end{cases}
$$

(2) If $\Gamma^{\prime}$ is a shifted complex on $[n]$ such that $f(\Gamma)=f\left(\Gamma^{\prime}\right)$, then $\Gamma=\Gamma^{\prime}$. (In other words every USLI complex is the only shifted complex in its $f$-class).

Proof: We verify part (1) by induction on $n+d+\sum k_{i}$. The assertion clearly holds if $d=1$ or if $\sum k_{i}=1$. For instance, if $d=1$ and $k_{1}=n$ (equivalently, $R_{1}=n+1$ ), then $F_{1}=[n+1, n]=\emptyset$ is the only facet of $\Gamma$.

Note that $R_{d}$ is the index of the first variable that does not divide any of the minimal generators of $I_{\Gamma}$. Thus if $R_{d} \leq n$, then $\Gamma=\mathrm{lk}_{\Gamma}(n) \star\{n\}$, and we are done by applying induction hypothesis to the USLI complex $\mathrm{lk}_{\Gamma}(n)$. So assume that $R_{d}=n+1$. Then $\mathrm{lk}_{\Gamma}(n)$ and ast $\Gamma_{\Gamma}(n)$ are easily seen to be the USLI complexes on the vertex set $[n-1]$ whose Stanley-Reisner ideals are given by $L_{1}=L\left(k_{1}, \ldots, k_{d-2}, k_{d-1}+1\right)$ and $L_{2}=L\left(k_{1}, \ldots, k_{d-1}, k_{d}-1\right)$, respectively. Hence by induction hypothesis the complex $\operatorname{lk}_{\Gamma}(n) \star\{n\}$ has exactly $d-1$ facets, namely the sets $F_{1}, \ldots, F_{d-1}$ from the list above. Now if $k_{d}>1$, then by induction hypothesis the facets of ast $\Gamma_{\Gamma}(n)$ are the sets $F_{1}-\{n\}, \ldots, F_{d-1}-\{n\}, F_{d}$. Since $\Gamma=\left(\mathrm{lk}_{\Gamma}(n) \star\{n\}\right) \cup \operatorname{ast}_{\Gamma}(n)$, it follows that $\max (\Gamma)=\left\{F_{1}, \ldots, F_{d}\right\}$. Similarly, if $k_{d}=$ 1 and $k_{j}$ is the last nonzero entry in the sequence $\left(k_{1}, \ldots, k_{d-1}\right)$, then the facets of ast $_{\Gamma}(n)$ are the sets $F_{1}-\{n\}, \ldots, F_{j-1}-\{n\}, F_{d}$, and the result follows in this case as well.

To prove part (2) we induct on $n$. The assertion is obvious for $n=1$. For $n>1$ we consider two cases.

Case 1: $R_{d} \leq n$. In this case $\Gamma=1 \mathrm{k}_{\Gamma}(n) \star\{n\}$, so $\beta_{i}(\Gamma)=0$ for all $i$. Since among all squarefree strongly stable ideals with the same Hilbert function the squarefree lexsegment ideal has the largest algebraic Betti numbers [2, Thm. 4.4], and since by Hochster's formula [12], $\beta_{n-i-1}(\Lambda)=\beta_{i-1, n}\left(I_{\Lambda}\right)$ for any simplicial complex $\Lambda$ on the vertex set [ $n$ ], it follows that $\beta_{i}\left(\Gamma^{\prime}\right) \leq \beta_{i}(\Gamma)=0$, and so $\beta_{i}\left(\Gamma^{\prime}\right)=0$ for all $i$. Since $\Gamma^{\prime}$ is shifted, Lemma 2.1 implies that all facets of $\Gamma^{\prime}$ contain $n$. Thus $\Gamma^{\prime}=\mathrm{lk}_{\Gamma^{\prime}}(n) \star\{n\}$, and the assertion follows from induction hypothesis applied to $\mathrm{lk}_{\Gamma}(n)$ and $\mathrm{lk}_{\Gamma^{\prime}}(n)$. 
Case 2: $R_{d}=n+1$. In this case all facets of $\Gamma$ but $F_{d}$ contain vertex $n$ (this follows from part (1) of the Lemma), and we infer from Lemma 2.1 that

$$
\beta_{i}(\Gamma)= \begin{cases}0, & \text { if } i \neq d-2 \\ 1, & \text { if } i=d-2\end{cases}
$$

Recall the Euler-Poincaré formula asserting that for any simplicial complex $\Lambda$,

$$
\sum_{j \geq-1}(-1)^{j} f_{j}(\Lambda)=\sum_{j \geq-1}(-1)^{j} \beta_{j}(\Lambda)=: \tilde{\chi}(\Lambda) .
$$

Therefore, $\quad \tilde{\chi}\left(\Gamma^{\prime}\right)=\sum_{j \geq-1}(-1)^{j} f_{j}\left(\Gamma^{\prime}\right)=\sum_{j \geq-1}(-1)^{j} f_{j}(\Gamma)=\tilde{\chi}(\Gamma)=(-1)^{d-2}, \quad$ and hence not all Betti numbers of $\Gamma^{\prime}$ vanish. The same reasoning as in Case 1 then shows that $\beta_{i}\left(\Gamma^{\prime}\right)=\beta_{i}(\Gamma)$ for all $i$. Applying Lemma 2.1 once again, we obtain that $\Gamma^{\prime}=\left(\mathrm{lk}_{\Gamma^{\prime}}(n) \star\{n\}\right) \cup\left\{F^{\prime}\right\}$, where $\left|F^{\prime}\right|=d-1$ and $F^{\prime}$ is the only facet of $\Gamma^{\prime}$ that does not contain $n$. Thus $f\left(\mathrm{lk}_{\Gamma}(n)\right)=f\left(\mathrm{lk}_{\Gamma^{\prime}}(n)\right)$ and $f\left(\operatorname{ast}_{\Gamma}(n)\right)=f\left(\operatorname{ast}_{\Gamma^{\prime}}(n)\right)$, and so $\operatorname{lk}_{\Gamma}(n)=\mathrm{lk}_{\Gamma^{\prime}}(n)$ and $\operatorname{ast}_{\Gamma}(n)=\operatorname{ast}_{\Gamma^{\prime}}(n)$ (by induction hypothesis), yielding that $\Gamma=\Gamma^{\prime}$.

We now turn to the class of almost USLIs. (Recall our convention that lower degree monomials are lex-larger than higher degree monomials.)

Definition 4.4. Let $I \subset S$ (or $I \subset S_{[n]}$ ) be a squarefree strongly stable monomial ideal with $G(I)=\left\{m_{1}>_{\text {lex }} \cdots>_{\text {lex }} m_{l}>_{\operatorname{lex}} m_{l+1}\right\}$. We say that $I$ is an almost USLI if $I$ is not a USLI, but $L=\left\langle m_{1}, \ldots, m_{l}\right\rangle$ is a USLI. We say that a simplicial complex $\Gamma$ is an almost USLI complex if $I_{\Gamma}$ is an almost USLI.

As we will see in the next section (see also Lemma 4.6 below), what makes almost USLI complexes noninvariant under lex shifting is the following combinatorial property. (We recall that the regularity of a finitely generated stable monomial ideal $I, \operatorname{reg}(I)$, is the maximal degree of its minimal generators.)

Lemma 4.5. Let $\Gamma$ be an almost USLI complex. Then $|\max (\Gamma)|>\operatorname{reg}\left(I_{\Gamma}\right)$.

Proof: Assume $\Gamma$ is a simplicial complex on $[n]$ with $G\left(I_{\Gamma}\right)=\left\{m_{1}>_{\text {lex }} \cdots>_{\text {lex }} m_{l}>_{\text {lex }}\right.$ $\left.m_{l+1}\right\}$. We have to show that $|\max (\Gamma)|>\operatorname{deg}\left(m_{l+1}\right)=: d$. We verify this by induction on $d$. To simplify the notation assume without loss of generality that every singleton $\{i\} \subset[n]$ is a vertex of $\Gamma$ (equivalently, $I_{\Gamma}$ has no generators of degree 1 ). If there are generators of degree 1 then the proof given below can be modified by letting the index $R_{1}$ play the role of the index 1. As $I_{\Gamma}$ is an almost USLI, and so $\left\langle m_{1}, \ldots, m_{l}\right\rangle$ is a USLI, this leaves two possible cases:

Case 1: $m_{1}, \ldots, m_{l}$ are divisible by $x_{1}$, but $m_{l+1}$ is not divisible by $x_{1}$. Since $I_{\Gamma}$ is squarefree strongly stable, it follows that $m_{l+1}=\prod_{j=2}^{d+1} x_{j}$. In this case each set $F_{i}=[n]-\{1, i\}, i=$ $2, \ldots, d+1$, is a facet of $\Gamma$. (Indeed the product $\prod\left\{x_{j}: j \in F_{i}\right\}$ is not divisible by $m_{l+1}$, and it is also not divisible by $x_{1}$, and hence by $m_{1}, \ldots, m_{l}$, implying that $F_{i}$ is a face. To show that $F_{i}$ is a maximal face observe that $F_{i} \cup\{i\}$ contains the support of $m_{l+1}$, and hence is not a face, but then shiftedness of $\Gamma$ implies that neither is $F_{i} \cup\{1\}$.) Since there also should be 
a facet containing 1 , we conclude that $\max (\Gamma) \geq d+1>\operatorname{deg}\left(m_{l+1}\right)$, completing the proof of this case.

Case 2: All minimal generators of $I$ are divisible by $x_{1}$. In this case consider an almost USLI $I_{\Gamma}^{\prime}:=\left\langle x_{1}, m_{1} / x_{1}, \ldots, m_{l+1} / x_{1}\right\rangle$. By induction hypothesis $\Gamma^{\prime}$ has $s>$ $\operatorname{deg}\left(m_{l+1}\right)-1$ facets which we denote by $F_{1}, \ldots, F_{s}$. One easily verifies that $\max (\Gamma)=$ $\left\{\{1\} \cup F_{1}, \ldots,\{1\} \cup F_{s},[2, n]\right\}$, and so $|\max (\Gamma)|=s+1>\operatorname{deg}\left(m_{l+1}\right)$.

We close this section with an algebraic lemma that relates regularity of $\operatorname{Gin}_{\text {lex }}\left(I_{\Gamma}\right)$ to the number of facets of $\Gamma$ (for an arbitrary complex $\Gamma$ ).

Lemma 4.6. For a (finite) simplicial complex $\Gamma, \operatorname{reg}\left(\operatorname{Gin}_{\text {lex }}\left(I_{\Gamma}\right)\right) \geq|\max (\Gamma)|$.

Proof: This fact is a corollary of [17, Lemma 23] applied to squarefree (and hence radical) ideal $I_{\Gamma} \in S_{[n]}$. For $\sigma \subseteq[n]$, we denote by $P_{\sigma}$ the (prime) ideal in $S_{[n]}$ generated by $\left\{x_{j}\right.$ : $j \notin \sigma\}$. It is well known that $I_{\Gamma}$ has the following prime decomposition: $I_{\Gamma}=\cap_{\sigma \in \max (\Gamma)} P_{\sigma}$. Thus the variety of $I_{\Gamma}, \mathcal{V}\left(I_{\Gamma}\right)$, is the union (over $\sigma \in \max (\Gamma)$ ) of the irreducible subvarieties $\mathcal{V}\left(P_{\sigma}\right)$. Each such subvariety is a linear subspace of $\mathbf{k}^{n}$ of codimension $n-|\sigma|$. [17, Lemma $23]$ then implies that the monomial $m:=\prod x_{i}^{r_{i}}$, where $r_{i}=|\{\sigma \in \max (\Gamma):|\sigma|=n-i\}|$, is a minimal generator of $\operatorname{Gin}_{\text {lex }}\left(I_{\Gamma}\right)$. Hence $\operatorname{reg}\left(\operatorname{Gin}_{\text {lex }}\left(I_{\Gamma}\right)\right) \geq \operatorname{deg}(m)=|\max (\Gamma)|$.

\section{Lex shifting, $B$-numbers and the limit complex}

In this section after defining the notion of lexicographic shifting and the notion of $B$-numbers (a certain analog of the Hilbert function) we prove Theorem 1.2. We remark that extending the notion of algebraic shifting to an arbitrary term order $\succ$ is not entirely automatic since the $\Phi$-image of the set of minimal generators of $\operatorname{Gin}_{\succ}\left(I_{\Gamma}\right) \subset S_{[n]}, G\left(\operatorname{Gin}_{\succ}\left(I_{\Gamma}\right)\right)$, may not be a subset of $S_{[n]}$. This however can be easily corrected if one considers the system of rings $S_{[n]}, n \in \mathbb{N}$, endowed with natural embeddings $S_{[n]} \subseteq S_{[m]}$ for $m \geq n$, and makes all the computations in the direct limit ring $S=\lim _{n \rightarrow \infty} S_{[n]}=\mathbf{k}\left[x_{i}: i \in \mathbb{N}\right]$. This is the approach we adopt here. We work with the class of monomial ideals $I \subset S$ finitely generated in each degree. Throughout this section we use the graded lexicographic term order on $S$.

Definition 5.1. Let $I$ be a monomial ideal of $S$ that is finitely generated in each degree. Define

$$
\operatorname{Gin}_{\text {lex }}(I):=\lim _{n \rightarrow \infty}\left(\operatorname{Gin}_{\text {lex }}\left(I \cap S_{[n]}\right)\right) S,
$$

where we consider $I \cap S_{[n]}$ as an ideal of $S_{[n]}$.

Since the $d$-th component of $\operatorname{Gin}_{\text {lex }}\left(I \cap S_{[n]}\right)$ depends only on the $d$-th component of $I \cap$ $S_{[n]}$, or equivalently on the minimal generators of $I \cap S_{[n]}$ of degree $\leq d$, Lemma 3.3(4) implies that $\operatorname{Gin}_{\text {lex }}(I)$ is well-defined and that for every $d$ there is $n(d)$ such that $\left(\operatorname{Gin}_{\text {lex }} I\right)_{d}=$ $\left(\left(\operatorname{Gin}_{\text {lex }}\left(I \cap S_{[n]}\right)\right) S\right)_{d}$ for all $n \geq n(d)$. Thus $\operatorname{Gin}_{\text {lex }}(I)$ is a monomial ideal finitely generated in each degree. (It is finitely generated if $I$ is.) Moreover, it follows from Lemma 3.3(1) that $\operatorname{Gin}_{\text {lex }}(I)$ is a strongly stable ideal.

Recall that the squarefree operation $\Phi$ takes monomials of $S$ to squarefree monomials of $S$. If $I \subset S$ is a monomial ideal finitely generated in each degree, we define $\Phi(I):=\langle\Phi(m)$ : $m \in G(I)\rangle$, where $G(I)$ is the set of minimal generators of $I$. 
Definition 5.2. Let $I$ be a homogeneous ideal of $S$ that is finitely generated in each degree. The lexicographic shifting of $I$ is the squarefree strongly stable ideal $\Delta_{\text {lex }}(I)=$ $\Phi\left(\operatorname{Gin}_{\text {lex }}(I)\right)$. The $i$-th lexicographic shifting of $I$ is the ideal $\Delta_{\text {lex }}^{i}(I)$, where $\Delta_{\text {lex }}^{i}$ stands for $i$ successive applications of $\Delta_{\text {lex }}$. We also define the limit ideal $\bar{\Delta}(I)$ : $=\lim _{k \rightarrow \infty} \Delta_{\text {lex }}^{k}(I)$.

The rest of the section is devoted to the proof of Theorem 1.2. First however we digress and review several facts on algebraic Betti numbers (defined by Eq. (2)).

Lemma 5.3. Let $I$ and $J$ be monomial ideals of $S_{[n]}$.

(1) If $I_{j}=J_{j}$ for all $0 \leq j \leq j_{0}$, then $\beta_{i, j}(I)=\beta_{i, j}(J)$ for all $i$ and all $j \leq j_{0}$.

(2) The Betti numbers of $I \subset S_{[n]}$ coincide with those of $I S_{[n+1]} \subset S_{[n+1]}$, that is, $\beta_{i, j}(I)=$ $\beta_{i, j}\left(I S_{[n+1]}\right)$ for all $i, j$.

Proof: Part (1) follows from the standard facts that

$$
\beta_{i, j}(I)=\operatorname{dim}_{\mathbf{k}} \operatorname{Tor}_{i}^{S_{[n]}}(\mathbf{k}, I)_{j}=\operatorname{dim}_{\mathbf{k}} \operatorname{Tor}_{i}^{S_{[n]}}(I, \mathbf{k})_{j},
$$

where we identify $\mathbf{k}$ with the $S_{[n]}$-module $S_{[n]} /\left\langle x_{1}, \ldots, x_{n}\right\rangle$. For part (2) note that if $\mathbb{F}$ is the free minimal resolution of $I$ over $S_{[n]}$, then $\mathbb{F} \otimes_{S_{[n]}} S_{[n+1]}$ is the free minimal resolution of $I S_{[n+1]}$ over $S_{[n+1]}$, yielding the lemma.

The above properties allow to extend the definition of the Betti numbers to the class of monomial ideals of $S$ that are finitely generated in each degree.

Definition 5.4. Let $I \subset S$ be a monomial ideal finitely generated in each degree. Define

$$
\beta_{i, j}(I):=\lim _{n \rightarrow \infty} \beta_{i, j}\left(I \cap S_{[n]}\right) \quad \text { for all } i, j \geq 0
$$

where we consider $I \cap S_{[n]}$ as an ideal of $S_{[n]}$.

We remark that since $I$ is finitely generated in each degree, for a fixed $j_{0}$ there exists $n_{0}$ such that $\left(I \cap S_{[n+1]}\right)_{j}=\left(\left(I \cap S_{[n]}\right) S_{[n+1]}\right)_{j}$ for all $0 \leq j \leq j_{0}$ and $n \geq n_{0}$. Hence it follows from Lemma 5.3 that (for a fixed $i$ ) the sequence $\left\{\beta_{i, j_{0}}\left(I \cap S_{[n]}\right)\right\}_{n \in \mathbb{N}}$ is a constant for indices starting with $n_{0}$, and thus $\beta_{i, j_{0}}(I)$ is well-defined.

The Betti numbers of strongly stable ideals (of $S_{[n]}$ ) were computed by Eliahou and Kervaire [10], and the analog of this formula for squarefree strongly stable ideals (of $S_{[n]}$ ) was established by Aramova et al. [2]. Definition 5.4 allows to state these results as follows. (For a monomial $u$ define $m(u):=\max \left\{i: x_{i} \mid u\right\}$.)

Lemma 5.5. Let $I \subset S$ be a monomial ideal finitely generated in each degree, let $G(I)$ denote its set of minimal generators, and let $G(I)_{j}=\{u \in G(I): \operatorname{deg} u=j\}$.

(1) If I is strongly stable, then $\beta_{i, i+j}(I)=\sum_{u \in G(I)_{j}}\left(\begin{array}{c}m(u)-1 \\ i\end{array}\right)$;

(2) If I is squarefree strongly stable, then $\beta_{i, i+j}(I)=\sum_{u \in G(I)_{j}}\left(\begin{array}{c}m(u)-j \\ i\end{array}\right)$. In particular, if $I=L\left(k_{\bullet}\right)$ is a USLI, then $\beta_{i, i+j}(I)=\sum_{l=1}^{k_{j}}\left(\begin{array}{c}k_{1}+\ldots+k_{j-1}+l-1 \\ i\end{array}\right)$. 
Using the notion of the Betti numbers, one can define a certain analog of the Hilbert function-the $B$-numbers-of a monomial ideal $I$ of $S$ that is finitely generated in each degree.

Definition 5.6. Let $I \subset S$ (or $I \subset S_{[n]}$ ) be a monomial ideal finitely generated in each degree, and let $\beta_{i, j}(I)$ be its graded Betti numbers. Define

$$
B_{j}(I):=\sum_{i=0}^{j}(-1)^{i} \beta_{i, j}(I) \quad \text { for all } j \geq 0 \quad\left(\text { e.g., } B_{0}=0 \text { and } B_{1}(I)=\left|G(I)_{1}\right|\right) \text {. }
$$

The sequence $B(I):=\left\{B_{j}(I): j \geq 1\right\}$ is called the $B$-sequence of $I$.

Remark 5.7. It is well known and is easy to prove (see [9, Section 1B.3]) that for every $n \in \mathbb{N}$ the polynomial $\sum_{j} B_{j}\left(I \cap S_{[n]}\right) x^{j}$ equals $(1-x)^{n} \operatorname{Hilb}\left(I \cap S_{n}, x\right)$, where $\operatorname{Hilb}\left(I \cap S_{n}, x\right)$ is the Hilbert series of $I \cap S_{[n]}$. In particular, if $\Gamma$ is a $(d-1)$-dimensional simplicial complex on $[n]$ and $I_{\Gamma} \subset S_{[n]}$ is its Stanley-Reisner ideal then

$$
\frac{1-\sum_{j} B_{j}\left(I_{\Gamma}\right) x^{j}}{(1-x)^{n}}=\operatorname{Hilb}\left(S_{[n]} / I_{\Gamma}, x\right)=\sum_{i=0}^{d} \frac{f_{i-1}(\Gamma) x^{i}}{(1-x)^{i}}=\frac{\sum_{i=0}^{d} h_{i}(\Gamma) x^{i}}{(1-x)^{d}},
$$

where $\left\{h_{i}(\Gamma)\right\}_{i=0}^{d}$ is the $h$-vector of $\Gamma$ [18]. (Recall that $h_{j}=\sum_{i=0}^{j}(-1)^{j-i}\left(\begin{array}{c}d-i \\ j-i\end{array}\right) f_{i-1}$ for $0 \leq j \leq d$. In particular, $h_{1}=f_{0}-d$.) Thus $\sum_{j} B_{j}\left(I_{\Gamma}\right) x^{j}=1-(1-x)^{h_{1}} \sum_{i} h_{i} x^{i}$ (if one assumes that $\{i\} \in \Gamma$ for every $i \in[n])$, and so the $h$-vector of $\Gamma$ defines the $B$-sequence of $I_{\Gamma}$.

The following lemma provides the analog of the " $f(\Gamma)=f\left(\Delta_{\mathrm{rl}}(\Gamma)\right)$-property".

Lemma 5.8. If I $\subset S$ is a monomial ideal that is finitely generated in each degree, then the ideals $I$ and $\Delta_{\text {lex }}(I)$ have the same $B$-sequence. In particular, if I is finitely generated, then for a sufficiently large $n$, the ideals $I \cap S_{[n]}$ and $\Delta_{\text {lex }}(I) \cap S_{[n]}$ have the same Hilbert function (in $\left.S_{[n]}\right)$.

Proof: Since for every $n \in \mathbb{N}$ the ideals $I \cap S_{[n]}$ and $\operatorname{Gin}_{\text {lex }}\left(I \cap S_{[n]}\right)$ have the same Hilbert function (in $S_{[n]}$ ) (see Lemma 3.3), and since $B_{i}(I)=\lim _{n \rightarrow \infty} B_{i}\left(I \cap S_{[n]}\right)$, the above remark implies that $B(I)=B\left(\operatorname{Gin}_{\text {lex }}(I)\right)$. Finally, since $\operatorname{Gin}_{\text {lex }}(I)$ is a strongly stable ideal (Lemma 3.3), we infer (by comparing the two formulas of Lemma 5.5) that $\beta_{i, j}\left(\operatorname{Gin}_{\text {lex }}(I)\right)=$ $\beta_{i, j}\left(\Phi \operatorname{Gin}_{\text {lex }}(I)\right)=\beta_{i, j}\left(\Delta_{\text {lex }}(I)\right)$ for all $i, j$, and so $B\left(\operatorname{Gin}_{\text {lex }}(I)\right)=B\left(\Delta_{\text {lex }}(I)\right)$. The result follows.

Now we are ready to verify the first part of Theorem 1.2. In fact we prove the following slightly more general result.

Theorem 5.9. Let I be a squarefree strongly stable ideal of $S$ finitely generated in each degree. Then $\Delta_{\text {lex }}(I)>_{\text {lex }}$ I unless $I$ is a USLI in which case $\Delta_{\text {lex }}(I)=I$. Moreover if I is finitely generated and is not a USLI, then all ideals in the sequence $\left\{\Delta_{\text {lex }}^{i}(I)\right\}_{i \geq 0}$ are distinct. 
Proof: There are several possible cases.

Case 1. $I=L\left(k_{\bullet}\right)$ is a USLI. To prove that $\Delta_{\text {lex }}(I)=I$, it suffices to show that for every $d \geq$ $1, \Delta_{\text {lex }}\left(L\left(k^{(d)}\right)=L\left(k^{(d)}\right)\right.$, where $k^{(d)}:=\left\{k_{1}, \ldots, k_{d}, 0,0, \ldots\right\}$ is the sequence $k_{\bullet}$ truncated at $k_{d}$. But this is immediate from Lemmas 4.3(2) and 5.8. Indeed, for $n=n(d)$ sufficiently large the simplicial complexes on the vertex set $[n]$ whose Stanley-Reisner ideals are given by $\Delta_{\text {lex }}\left(L\left(k^{(d)}\right) \cap S_{[n]}\right.$ and $L\left(k^{(d)}\right) \cap S_{[n]}$, respectively, are shifted and have the same $f$-numbers. Since the second complex is a USLI complex, it follows that those complexes, and hence their ideals, coincide.

Case 2. $I=\left\langle m_{1}, \ldots, m_{l}, m_{l+1}\right\rangle$ is an almost USLI. Let $n$ be the largest index of a variable appearing in $\prod_{i=1}^{l+1} m_{i}$, and let $\Gamma$ be a simplicial complex on $[n]$ with $I_{\Gamma}=I \cap S_{[n]}$. Then

$$
\operatorname{reg}\left(\Delta_{\text {lex }}(I)\right)=\operatorname{reg}\left(\operatorname{Gin}_{\text {lex }}\left(I_{\Gamma}\right)\right) \stackrel{\text { Lemma } 4.6}{\geq}|\max (\Gamma)| \stackrel{\text { Lemma } 4.5}{>} \operatorname{reg}\left(I_{\Gamma}\right)=\operatorname{reg}(I),
$$

yielding that $\Delta_{\text {lex }}(I) \neq I$ in this case. Moreover, since by Eq. (1), $\Phi\left(\operatorname{Gin}_{\mathrm{rl}}\left(I_{\Gamma}\right)\right)=I_{\Gamma}$ and since $\Phi$ is a lex-order preserving map, we infer from Lemma 3.5 that $\Phi\left(\operatorname{Gin}_{\text {lex }}\left(I_{\Gamma}\right)\right) \geq_{\text {lex }}$ $\Phi\left(\operatorname{Gin}_{\mathrm{rl}}\left(I_{\Gamma}\right)\right)=I_{\Gamma}$, and hence that $\Delta_{\text {lex }}(I)>_{\text {lex }} I$.

Case 3. I is squarefree strongly stable, but is not a USLI. In this case we sort $G(I)=$ $\left\{m_{1}, \ldots, m_{l}, m_{l+1}, \cdots\right\}$ by graded lex-order and assume that $m_{l+1}$ is the first non-USLI generator of $I$. Let $I_{1}=\left\langle m_{1}, \ldots, m_{l}\right\rangle$ and let $I_{2}=\left\langle m_{1}, \ldots, m_{l+1}\right\rangle$. Then $I_{1}$ is a USLI, $I_{2}$ is an almost USLI, and $I_{1} \subset I_{2} \subseteq I$. Hence by the previous two cases $I_{1}=\Delta_{\text {lex }}\left(I_{1}\right) \subset \Delta_{\text {lex }}\left(I_{2}\right)$ and $\Delta_{\text {lex }}\left(I_{2}\right)>_{\text {lex }} I_{2}$, and so there exists a monomial $m, m_{l}>_{\text {lex }} m>_{\text {lex }} m_{l+1}$, such that $m \in$ $G\left(\Delta_{\text {lex }}\left(I_{2}\right)\right) \subseteq G\left(\Delta_{\text {lex }}(I)\right)$. Thus $\Delta_{\text {lex }}(I)>_{\text {lex }} I$.

Finally to show that for a finitely generated ideal $I$, all ideals in the sequence $\left\{\Delta_{\text {lex }}^{i}(I)\right\}_{i \geq 0}$ are distinct, it suffices to check that none of those ideals is a USLI. This is an immediate corollary of Lemmas 4.3(2) and 5.8.

Our next goal is to prove the second part of Theorem 1.2. To do that we fix a sequence of integers $B=\left\{B_{j}: j \geq 1\right\}$ and study the class $\mathcal{M}(B)$ of all monomial ideals $I \subset S$ that are finitely generated in each degree and satisfy $B(I)=B$.

Lemma 5.10. There is at most one USLI in the class $\mathcal{M}(B)$.

Proof: Recall that a USLI $L=L\left(k_{\bullet}\right)$ is uniquely defined by its $k$-sequence $k_{\bullet}=\left\{k_{i}: i \geq 1\right\}$, where $k_{i}=\beta_{0, i}(L)=\left|G(L)_{i}\right|$. Recall also that $B(L)$ is a function of $k_{\bullet}$ (see Lemma $5.5(2)$ ), and so to complete the proof it suffices to show that this function is oneto-one, or more precisely that $k_{j}$ is determined by $k_{1}, \ldots, k_{j-1}, B_{j}$ (for every $j \geq 1$ ). And indeed,

$$
\begin{aligned}
k_{j} & \left.=\beta_{0, j}(L)=B_{j}-\sum_{i=1}^{j}(-1)^{i} \beta_{i, j}(L) \quad \text { (by definition of } B_{j}\right) \\
& =B_{j}-\sum_{i=1}^{j}(-1)^{i} \sum_{l=1}^{k_{j-i}}\left(\begin{array}{c}
k_{1}+\ldots+k_{j-i-1}+l-1 \\
i
\end{array}\right) \quad \text { (by Lemma 5.5(2)). }
\end{aligned}
$$

Now we are ready to prove (the slightly more general version of) the second part of Theorem 1.2. 
Theorem 5.11. For every ideal $I \in \mathcal{M}(B)$, the limit ideal $\bar{\Delta}_{\text {lex }}(I)$ is well defined and is the unique USLI of $\mathcal{M}(B)$.

Proof: Fix $I \in \mathcal{M}(B)$. To show that $\bar{\Delta}_{\text {lex }}(I)$ is well defined, it suffices to check that for every $d \geq 0$, there exists $s=s(d)$ such that

$$
G\left(\Delta_{\text {lex }}^{s}(I)\right)_{\leq d}=G\left(\Delta_{\text {lex }}^{s+1}(I)\right)_{\leq d}
$$

(where $\left.G(J)_{\leq d}:=\cup_{j \leq d} G(J)_{j}\right)$, and hence that all ideals $\Delta_{\text {lex }}^{i}(I), i \geq s$, have the same $d$-th homogeneous component. We verify this fact by showing that the collection of all possible sets of minimal generators

$$
\mathcal{G}_{\leq d}:=\left\{G(J)_{\leq d}: J \in \mathcal{M}(B), J \text { is squarefree strongly stable }\right\} \text { is finite. }
$$

(This yields (3), since all ideals $\Delta_{\text {lex }}^{i}(I), i \geq 1$, are squarefree strongly stable, and since $\Delta_{\text {lex }}^{i}(I) \leq_{\text {lex }} \Delta_{\text {lex }}^{i+1}(I)$ by Theorem 5.9.) Eq. (4) can be easily proved by induction. It clearly holds for $d=0$. Now if $J \in \mathcal{M}(B)$ is squarefree strongly stable, then by Lemma 5.5(2) and Definition 5.6,

$$
\left|G(J)_{d}\right|=\beta_{0, d}(J)=B_{d}-\sum_{i=1}^{d}(-1)^{i} \sum_{u \in G(J)_{d-i}}\left(\begin{array}{c}
m(u)-(d-i) \\
i
\end{array}\right)
$$

so assuming that the collection $\mathcal{G}_{\leq d-1}$ is finite, or equivalently that the set of integers $\{m(u)$ : $\left.u \in G(J)_{\leq d-1} \in \mathcal{G}_{\leq d-1}\right\}$ is bounded (say by $n(d)$ ), we obtain that there exists a constant $g(d)$ such that $\left|G(J)_{d}\right| \leq g(d)$ for all squarefree strongly stable ideals $J \in \mathcal{M}(B)$. But then the squarefree strongly stable property implies that $m(u)<n(d)+g(d)+d$ for every $u \in$ $G(J)_{\leq d} \in \mathcal{G}_{\leq d}$, and (4) follows.

The second part of the statement is now immediate: indeed if $G\left(\Delta^{s}(I)\right)_{\leq d}=$ $G\left(\Delta^{s+1}(I)\right)_{\leq d}$, then by Theorem $5.9, G\left(\Delta^{s}(I)\right)_{\leq d}=G(\bar{\Delta}(I))_{\leq d}$ is the set of minimal generators of a USLI.

\section{Remarks on other term orders}

We close the paper by discussing several results and conjectures related to algebraic shifting with respect to arbitrary term orders. To this end, we say that an order $\succ$ on monomials of $S$ is a term order if $x_{i} \succ x_{i+1}$ for $i \geq 1, m \succ m^{\prime}$ as long as $\operatorname{deg}(m)<\operatorname{deg}\left(m^{\prime}\right)$, and the restriction of $\succ$ to $S_{[n]}$ is a term order on $S_{[n]}$ for all $n \geq 1$. In addition, we restrict our discussion only to those term orders on $S$ that are compatible with the squarefree operation $\Phi$, that is, $\Phi(m) \succ \Phi\left(m^{\prime}\right)$ if $m \succ m^{\prime}$.

Similarly to Definition 5.1, for a term order $\succ$ on $S$ and a homogeneous ideal $I \subset S$ that is finitely generated in each degree, we define $\Delta_{\succ}(I):=\Phi\left(\operatorname{Gin}_{\succ}(I)\right)$. Thus $\Delta_{\succ}(I)$ is a squarefree strongly stable ideal that has the same $B$-sequence as $I$. (Indeed, the proof of Lemma 5.8 carries over to this more general case.)

We say that a squarefree monomial ideal $I \subset S$ is a $U S \succ I$ if for every monomial $m \in I$ and every squarefree monomial $m^{\prime}$ such that $\operatorname{deg}(m)=\operatorname{deg}\left(m^{\prime}\right)$ and $m^{\prime} \succ m, m^{\prime}$ is an element of $I$ as well. Being US $\succ I$ implies being squarefree strongly stable.

In view of Theorems 1.2 and 3.7 it is natural to ask the following: 
(1) Does $\Delta_{\succ}(I)=I$ hold for every US $\succ$ I I?

(2) Is there a term order $\succ$ other than the lexicographic order for which the equality $\Delta_{\succ}(I)=I$ implies that $I$ is a $\mathrm{US} \succ \mathrm{I}$ ?

(3) Is there a term order $\succ$ other than the reverse lexicographic order such that the equation $\Delta_{\succ}(I)=I$ holds for all squarefree strongly stable ideals $I$ ?

The next proposition answers the first question in the affirmative.

Proposition 6.1. If I is a $U S \succ I$, then $\Delta_{\succ}(I)=$ I for every term order on $S$ that is compatible with $\Phi$.

Proof: Exactly as in the proof of Theorem 5.9 (see the last three lines of Case 2), one can show that $\Delta_{\succ}(I) \succeq I$. Hence either $\Delta_{\succ}(I)=I$, in which case we are done, or the $\succ$-largest monomial, $m$, in the symmetric difference of $G\left(\Delta_{\succ}(I)\right)$ and $G(I)$ is an element of $G\left(\Delta_{\succ}(I)\right)$. Since $I$ is a $\mathrm{US} \succ \mathrm{I}$, we obtain in the latter case that $G\left(\Delta_{\succ}(I)\right)_{i}=G(I)_{i}$ for all $i<\operatorname{deg}(m)$ and

$$
G(I)_{i_{0}}=\left\{m^{\prime} \in G\left(\Delta_{\succ}(I)\right)_{i_{0}}: m^{\prime} \succ m\right\} \quad \text { for } i_{0}=\operatorname{deg}(m),
$$

that is, $G(I)_{i_{0}}$ is a strict subset of $G\left(\Delta_{\succ}(I)\right)_{i_{0}}$. This is however impossible, since it contradicts the fact that the ideals $I$ and $\Delta_{\succ}(I)$ have the same $B$-sequence.

The answer to the second question is negative as follows from the following result.

Proposition 6.2. If I is a USLI, then $\Delta_{\succ}(I)=I$ for all term orders $\succ$.

We omit the proof as it is completely analogous to that of Theorem 5.9, Case 1.

While we do not know the answer to the third question, we believe that it is negative. In fact it is tempting to conjecture that the following holds. Let $>$ be a term order on $S$ other than the (graded) reverse lexicographic order, and let $k \geq 2$ be the smallest degree on which $\succ$ and revlex disagree. Write $m_{i}$ to denote the $i$ th squarefree monomial of $S$ of degree $k$ with respect to the revlex order. (It is a fundamental property of the revlex order that every squarefree monomial of $S$ of degree $k$ is of the form $m_{i}$ for some finite $i$.)

Conjecture 6.1. Let $i_{0} \geq 1$ be the smallest index for which $I_{i_{0}}:=\left\langle m_{1}, \ldots, m_{i_{0}}\right\rangle$ is not a $\mathrm{US} \succ I$. Then $\Delta_{\succ}\left(I_{i_{0}}\right) \neq I_{i_{0}}$.

Acknowledgmnts We are grateful to Aldo Conca for helpful discussions and to the anonymous referees for insightful comments.

\section{References}

1. A. Aramova and J. Herzog, "Almost regular sequences and Betti numbers," American J. Math. 122 (2000), 689-719.

2. A. Aramova, J. Herzog, and T. Hibi, "Squarefree lexsegment ideals," Math. Z. 228 (1998), 353-378.

3. A. Aramova, J. Herzog, and T. Hibi, "Shifting operations and graded Betti numbers," J. Algebraic Combin. 12(3), (2000), 207-222.

4. D. Bayer, H. Charalambous, and S. Popescu, "Extremal Betti numbers and applications to monomial ideals," J. Algebra 221(2), (1999), 497-512. 
5. A.M. Bigatti, A. Conca, and L. Robbiano, "Generic initial ideals and distractions," Comm. Algebra 33(6) (2005), 1709-1732.

6. A. Björner and G. Kalai, "An extended Euler-Poincare theorem," Acta Math. 161 (1988), 279-303.

7. A. Conca, "Reduction numbers and initial ideals," Proc. Amer. Math. Soc. 131 (2003), 1015-1020.

8. D. Eisenbud, Commutative Algebra with a View Toward Algebraic Geometry, Springer-Verlag GTM, New York, 1995.

9. D. Eisenbud, The Geometry of Syzygies, Springer-Verlag GTM, 2004, to appear.

10. S. Eliahou and M. Kervaire, "Minimal resolutions of some monomial ideals," J. Alg. 129 (1990), 1-25.

11. J. Herzog, "Generic initial ideals and graded Betti numbers," Computational Commutative Algebra and Combinatorics, (ed. T. Hibi), Vol. 33 of Advanced Studies in Pure Mathematics, Mathematical Society of Japan, Tokyo, 2002, pp. 75-120.

12. M. Hochster, "Cohen-Macaulay rings, combinatorics and simplicial complexes," Proc. Ring Theory II, Lect. Notes in Pure and Applied Math., 26, Dekker, New York, 1977, pp. 171-223.

13. G. Kalai, "Symmetric matroids," J. Combin. Theory Ser. B 50 (1990), 54-64.

14. G. Kalai, "The diameter of graphs of convex polytopes and f-vector theory," Applied Geometry and Discrete Mathematics - The Victor Klee Festschrift (eds: P. Gritzmann and B. Sturmfels), DIMACS Series in Discrete Mathematics and Theoretical Computer Science, Vol. 4, Amer. Math. Soc., Providence, RI, 1991, pp. 387-411.

15. G. Kalai, "Algebraic shifting," in Computational Commutative Algebra and Combinatorics, T. Hibi (ed.), Vol. 33 of Advanced Studies in Pure Mathematics, Mathematical Society of Japan, Tokyo, 2002, pp. 121163.

16. E. Nevo, "Algebraic shifting and basic constructions on simplicial complexes," preprint (2002).

17. K. Pardue, "Deformation classes of graded modules and maximal Betti numbers," Illinois J. Math 40 (1996), 564-585.

18. R. Stanley, Combinatorics and Commutative Algebra, Second Edition, Birkhäuser, Boston, 1996. 